\title{
LOCALIZATION OF LEFT-TO-RIGHT SHUNTS BY RADIOACTIVE KRYPTON
}

\author{
BY \\ DAVID WEITZMAN, JOAN MCALISTER, AND ANTHONY HOULDER \\ From the Departments of Cardiology and Radio-Isotopes, St. Bartholomew's Hospital
}

Received November 7, 1963

In 1958 Weitzman and McAlister reported on the use of a radioactive tracer $\left({ }^{32} \mathrm{P}\right)$ for accurate localization of the site of a left-to-right shunt in congenital cardiac lesions. The advantage of such a technique is that it can demonstrate premature arrival of the tracer in the chamber or vessel that receives the shunt. There is a clear-cut time difference due to the short circuit through the defect. This method obviates the difficulties of interpretation that can be caused by incomplete mixing when shunt diagnosis is based upon oxygen saturations.

The ${ }^{32} \mathrm{P}$ method has a number of disadvantages. The isotope has a physical half-life of 14.3 days, and a fairly long biological half-life: hence the test cannot easily be repeated in the course of a single catheterization. Two separate catheters (or a twin-lumen) have to be inserted for injection and sampling. A false positive can result from pulmonary valve incompetence (Weitzman and McAlister, 1958). The last two disadvantages are overcome by the use of a radioactive gas, as this can be introduced into the pulmonary circulation by inhalation. Sanders and Morrow (1959) utilized ${ }^{85} \mathrm{Kr}$ for this purpose. It has several advantages over other possible radioactive gases. First, it is an inert gas, and so has a very short biological half-life, being completely cleared from the body in the exhaled air within a few minutes. A fairly large dose can therefore be given without significant radiation risk; in addition, the investigation can be repeated after a very short time interval, thus eliminating the necessity for simultaneous sampling which means the use of a twin-lumen catheter. Secondly, it has a long physical half-life, so that it is easy to maintain a constant supply. Thirdly, it is almost a pure beta-emitter, which is an advantage from the point of view of exact time measurement. Sanders and Morrow compared concentrations of ${ }^{85} \mathrm{Kr}$ in pulmonary and systemic arterial samples for shunt diagnosis. We have already pointed out the preference for continuous sampling and a demonstrable time difference, and have therefore adapted the ${ }^{85} \mathrm{Kr}$ technique to that employed for the ${ }^{32} \mathrm{P}$.

\section{METHOD}

Dispensing Doses. The first problem was the administration of the krypton. We decided that as it was too great a risk to have a cylinder containing a curie or more of radioactive krypton in the catheter room, each individual dose must be dispensed previously. In order to save expense we decided to do this ourselves and designed a nearly all-metal system made in our own workshop. The equipment is shown in Fig. 1. The glass ampoule containing 500 millicuries of ${ }^{85} \mathrm{Kr}$ was connected by a metal-to-glass seal and the gas transferred to the storage chamber, the pressure being brought up to atmospheric with inactive krypton. The krypton is stored in this chamber and 10 millicurie doses are dispensed as required into one of the sampling tubes which may be connected by a standard vacuum union. The advantage of this over the more conventional glass apparatus is that no glass-blowing is required, and the procedure can be carried out by unskilled personnel. 


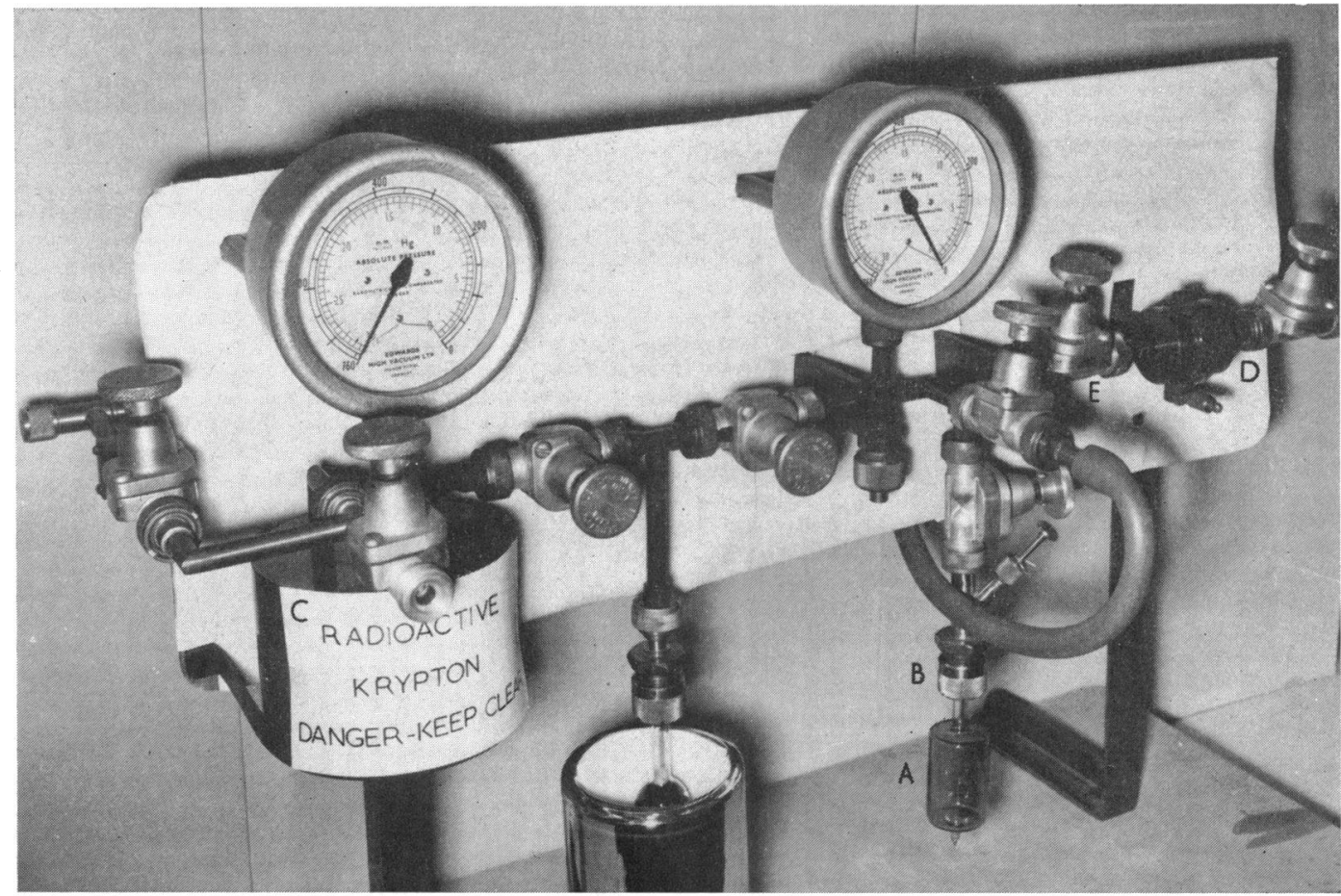

FIG. 1.- $\mathrm{A}=$ glass ampoule containing 500 millicuries of $85 \mathrm{Kr} ; \mathrm{B}=$ metal-to-glass seal; $\mathrm{C}=$ storage chamber; $\mathrm{D}=$ sampling tube; $\mathrm{E}=$ standard vacuum union and valve. The storage chamber $(\mathrm{C})$, marked "radioactive krypton," is at atmospheric pressure, as shown by the left-hand pressure gauge. The sampling tube (D), on the extreme right, has been evacuated ready for loading, as indicated by the right-hand pressure gauge.

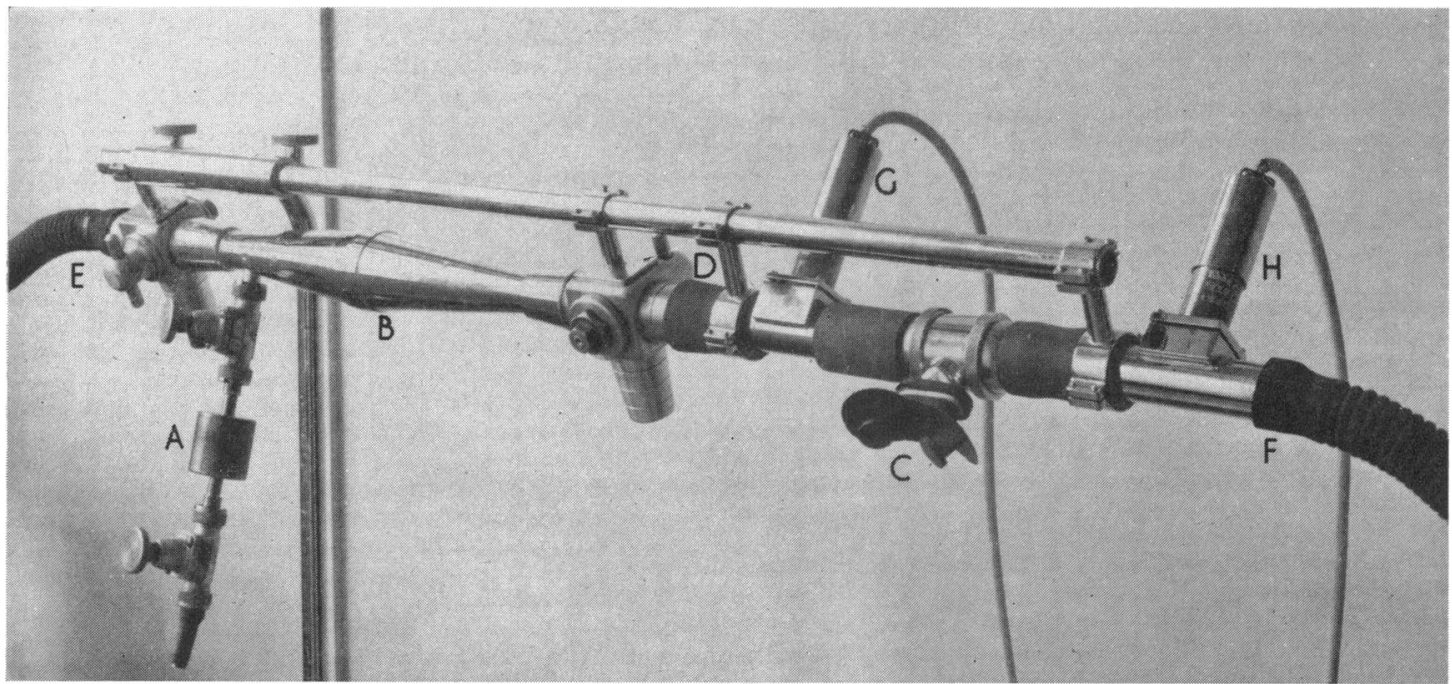

FIG. 2.-A=sampling tube; $\mathrm{B}=$ breathing tube; $\mathrm{C}=$ mouthpiece; $\mathrm{D}=\operatorname{tap} ; \mathrm{E}=$ connexion to spirometer; $F=$ connexion to Douglas bag; $G$ and $H=$ "inspiration" and "expiration" Geiger counter. The sampling tube (A) is shown connected to the breathing tube (B). Both taps on the breathing tube are shown closed (D and E), so that the mouthpiece is open to air. 


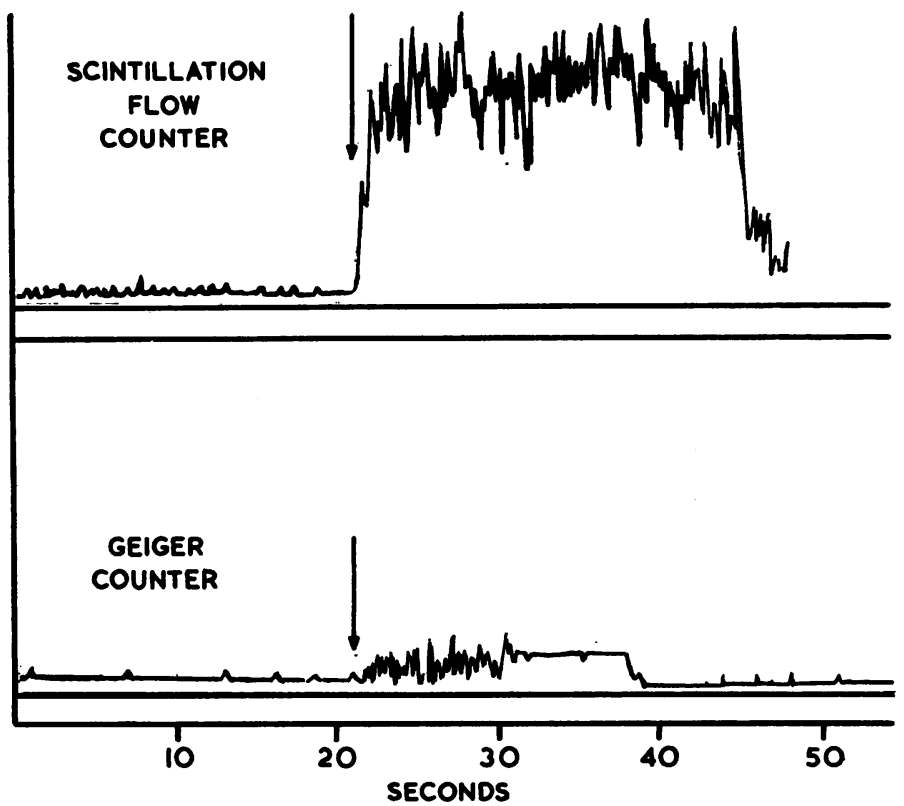

FIG. 3.-Recordings obtained with the same sample of $85 \mathrm{Kr}$-loaded blood, demonstrating that the sensitivity of the scintillation flow counter is about ten times that of the Geiger counter.

Administration of Krypton to the Patient. Since the essence of the technique is the detection of a very short time interval it is essential to get a sharp starting point. We therefore use a single-breath technique. A similar technique for administering radioactive krypton and methyl iodide has been used by Jaimet, Tomlinson, and Nace (1958). Our apparatus is shown in Fig. 2. Before the investigation, 10 millicuries of krypton are transferred from one of the sampling tubes to the breathing tube of volume $400 \mathrm{ml}$. and taken up to atmospheric pressure by diluting with air. When the mouthpiece is inserted in the mouth, the patient can breathe quietly in and out to air until everything is ready. When the tap is turned the patient inhales the krypton; as he breathes out, the tap is turned to its original position and he is again breathing air. A spirometer is connected to the afferent end of the breathing tube so that the breathing tube is well flushed out with air from the spirometer and the volume of an average deep inspiration is recorded. After the inhalation of the krypton, the expired breath is collected in a Douglas bag until its activity is insignificant, which is usually at five to ten minutes after inhalation.

Detection of the Radioactive Tracer in the Heart Chamber. In order to get maximum sensitivity we used a scintillation flow-counter for the detection of ${ }^{85} \mathrm{Kr}$ in the blood. The radiation emitted by ${ }^{85} \mathrm{Kr}$ is $99 \cdot 3$ per cent beta-radiation of maximum energy $0.67 \mathrm{MeV}$, and only 0.7 per cent gamma-radiation of energy 0.54 $\mathrm{MeV}$. The lower energy beta-radiation is easily absorbed, the intensity being reduced to one-half by 0.004" Al. In the scintillation flow-counter, the blood actually flows through, i.e. is in direct contact with, a coil of plastic phosphor, and there is no loss in efficiency due to absorption, apart from self-absorption.

Figure 3 shows a comparison of the sensitivity to krypton, of the scintillation counter, and the endwindow Geiger counter method used previously for phosphorus-32. The scintillation counter is about 10 times as efficient as the end-window counter.

\section{Procedure}

Figure 4 shows a schematic diagram of the complete apparatus. It consists of two main parts. One part is concerned with the inhalation of the krypton, with recording the times of inspiration and expiration, and with monitoring the expired breath. The breathing system has been described above. To record the time of inspiration, a Geiger counter was inserted into the system between the tube containing the krypton and the mouthpiece. This counter was connected to a ratemeter whose output was fed to the lower channel of a Sanborn "Twin-Viso" direct writing recorder. The paper of the recorder moves at a speed of $0 \cdot 25 \mathrm{~cm}$. 


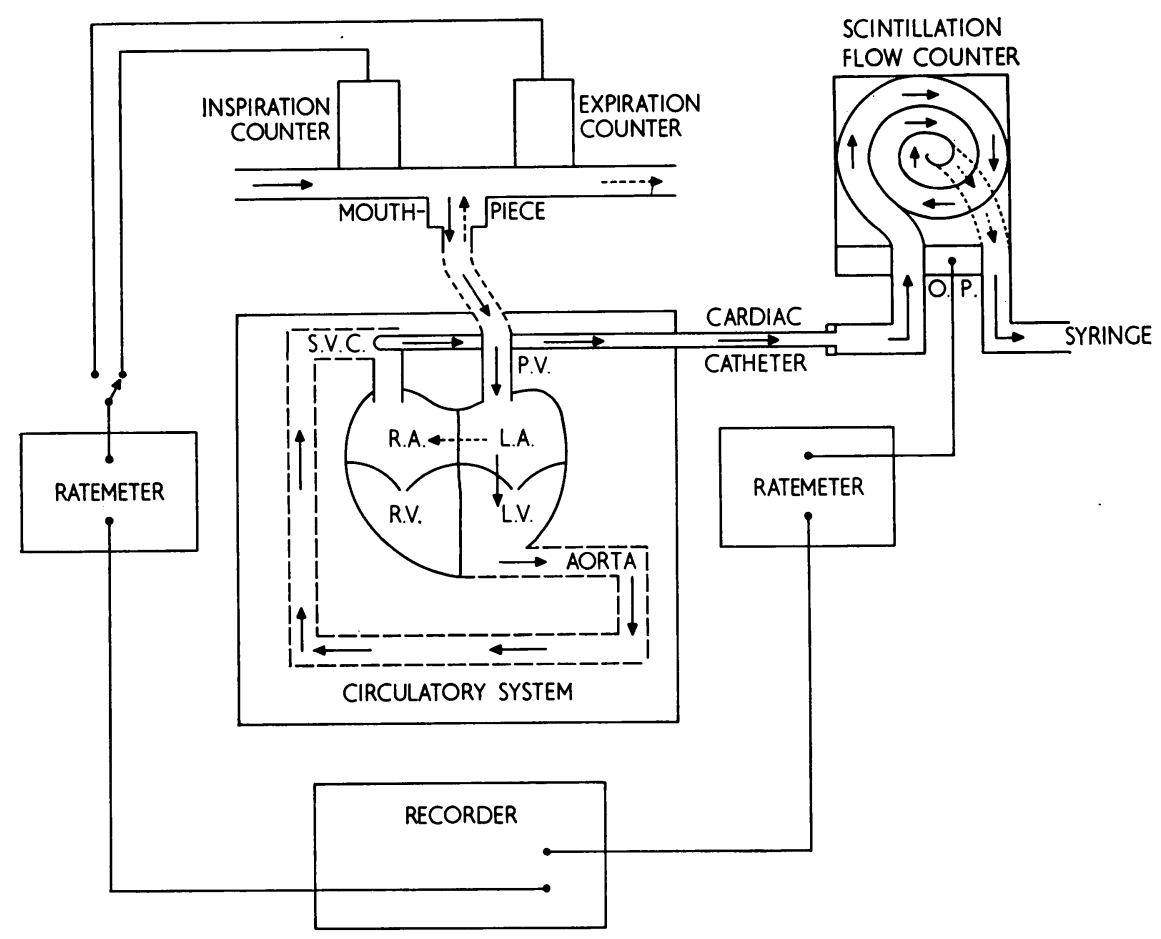

Fig. 4.-The passage of the $85 \mathrm{Kr}$ through the circulation. The full arrows demonstrate the normal route, and the one dotted arrow, from left to right atrium, shows the route in the case of an atrial septal defect.

per second. As the patient breathes in the krypton, the flow of radioactivity past the counter is recorded as a sharp peak on the lower channel of the recorder. Another counter was inserted on the efferent side of the mouthpiece, connected to a ratemeter whose output was switched to the lower channel, immediately after the "inhalation peak" was observed. As the patient exhales the krypton, a sharp rise is recorded on the lower channel, followed by a rhythmic decrease as the gas is rapidly cleared from the body.

The other part of the equipment is concerned with recording the appearance of the krypton in the "sampling" chamber. Blood is withdrawn from the chamber via the catheter and through the scintillation counter by a motor-driven syringe. The scintillation counter is connected to an amplifier and ratemeter whose output is fed into the upper channel of the recorder. The appearance of the radioactivity in the flowcounter is recorded as a rise on the upper channel.

The procedure is as follows. The cardiac catheter having been inserted with its tip in the appropriate heart chamber, the patient is left quietly breathing air through the mouthpiece. The catheter is connected to the motor-driven syringe via the flow-counter and the motor is started so that blood is withdrawn through the counter. Simultaneously the patient is instructed to breathe in, out, and in again: as he breathes out, the taps are turned so that on the second inspiration he inhales the krypton; at the next expiration the taps are returned to the original positions, so that the patient is again breathing air and continues to breathe normally. The times of inhalation of the gas, of exhalation, and of the subsequent decrease of activity in the expired breath, are recorded on the lower channel, and the time of appearance of radioactive krypton in the flowcounter is recorded on the upper channel. The time interval between inhalation of the krypton, and its appearance in the flow-counter is recorded exactly. There is, of course, a delay between the appearance of radioactivity in the sampling chamber and its appearance in the flow-counter, but this time can be measured. In practice, the diagnosis depends on the difference in times when sampling from two chambers, and therefore the delay time is unimportant so long as it is constant. 


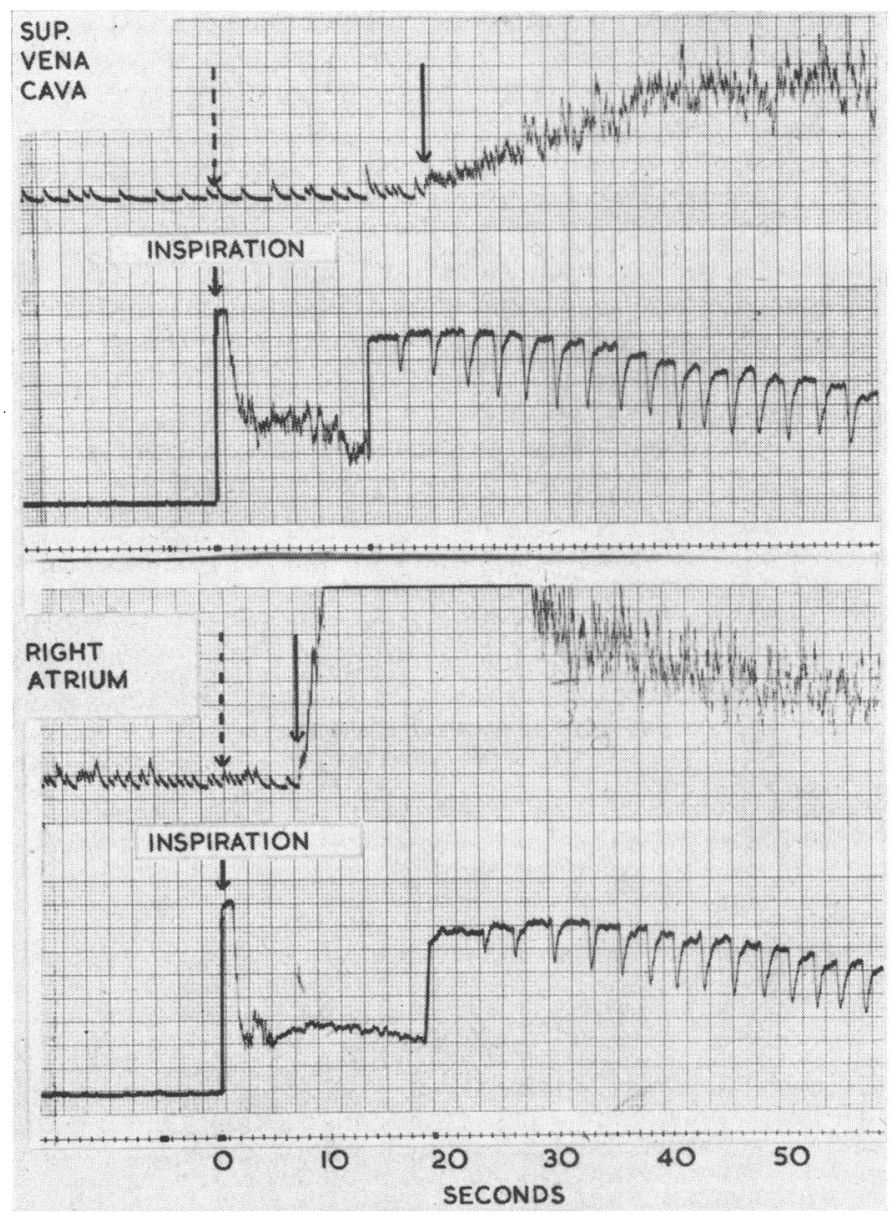

FIG. 5.-The top curves, sampling from superior vena cava, show an arrival time of 20 seconds (including superior vena cava to counter time of 3.6 seconds). The lower curves, sampling from right atrium, show a circulation time of 6.8 seconds (less the right atrium to counter time of 3.4 seconds).

\section{RESULTS}

Diagnostic krypton studies have been carried out in 16 patients. Fig. 5 is a recording of a case of atrial septal defect. The upper two recordings were obtained when sampling from the superior vena cava: the arrival time was 16.4 seconds. The lower two were obtained when sampling from the right atrium: the arrival time here was only 3.4 seconds. There is a difference between the two arrival times, unmistakably demonstrating a left-to-right shunt at atrial level. There is, too, an obvious difference in the shape of the curves; the slow rise of the caval tracing is due to venous blood arriving at different times from various parts of the body. The atrial tracing, which was purposely made on the same range, shows, in contrast, a very sharp rise demonstrating a rapid appearance from left atrium only, and a higher concentration due, of course, to less dilution. In this patient the oxygen saturation of caval blood was 12.26 volumes per cent, and that of the right atrial blood 13.96 volumes per cent. This is slightly below the accepted figure of 2 volumes per cent for a significant difference, and differences of this order can arise from poor mixing in the right atrium (Morrow, Sanders, and Braunwald, 1958).

Examples are given of other patients in whom diagnostic problems were solved by this technique. 
Case 1. A boy aged 15 had a loud parasternal systolic murmur. On clinical grounds, the diagnosis considered most likely was ventricular septal defect. On catheterization, oxygen saturations were as follows: pulmonary artery 80 and 78.5 per cent; high right ventricle 80 and 78 per cent; low right ventricle 78.5 and 74 per cent; right atrium 76.5 and 75 per cent; and superior vena cava 76 and 74.5 per cent. While suggestive, these figures do not show any significant differences. Inhaled ${ }^{85} \mathrm{Kr}$, however, appeared in the right ventricle in only $\mathbf{4}$ seconds, as compared with 19 seconds to reach the right atrium, confirming a left-to-right shunt at ventricular level.

Case 2. A girl aged 24 had clinical, radiological, and electrocardiographic evidence of a ventricular septal defect. This was apparently confirmed by routine catheterization, which showed oxygen saturations ranging between 83.5 and 86 per cent in right ventricle and pulmonary artery; and between 75.5 and 77.5 per cent in right atrium and superior vena cava. ${ }^{85} \mathrm{Kr}$ appeared in the right atrium only 3 seconds after inhalation, indicating a left-to-right shunt at atrial level. This finding, taken with the clinical features, suggested that a "Gerbode" type of defect might be present. This was confirmed at operation, there being a ventricular septal defect with a fenestrated septal cusp of the tricuspid valve.

Case 3. Catheter proof was sought of closure of the ventricular septal defect after surgical correction of Fallot's tetralogy in a boy aged 16. Oxygen saturations showed a scatter over 60 to 69 per cent: pulmonary artery 69,69 , and 68 per cent; right ventricle $63,63 \cdot 5$, and 66 per cent; right atrium 62,69 , and 65 per cent; and superior vena cava 60,62 , and 65 per cent. These show no conclusive differences, but the single right atrium figure of 69 per cent could well have been due to poor mixing: and, this excepted, pulmonary artery saturations are slightly up on proximal chambers, which would be compatible with a small high persistent ventricular septal defect. However, the arrival time of inhaled ${ }^{85} \mathrm{Kr}$ was 14 seconds in the right atrium and 12.4 seconds in pulmonary artery, disproving any short circuit. (In this patient, ${ }^{85} \mathrm{Kr}$ in solution was also injected via the catheter into the right atrium, following the technique described by Long, Braunwald, and Morrow (1960). No radioactivity could subsequently be detected in pulmonary arterial blood, confirming the absence of any right-to-left shunt. In the absence of such shunt, ${ }^{85} \mathrm{Kr}$ introduced into the right heart chambers is all excreted via the exhaled air.)

No untoward effects were encountered in any patient. In all subsequently operated cases, the ${ }^{85} \mathrm{Kr}$ findings were confirmed.

\section{DisCUSSION}

We have confirmed Sanders and Morrow's (1959) view that ${ }^{85} \mathrm{Kr}$ is a valuable and useful tracer for shunt diagnosis. Errors are known to arise from conventional methods of localization because of variations in oxygen content of mixed venous blood (Warren, Stead, and Brannon, 1946), and inefficient mixing of superior caval, inferior caval, coronary sinus, and shunt streams in the right atrium. Morrow et al. (1958) showed that such errors were far less frequent with nitrous oxide and ${ }^{85} \mathrm{Kr}$ (loc. cit.). With the comparison of concentrations of ${ }^{85} \mathrm{Kr}$ in pulmonary and systemic arteries an overlap still occurs between figures associated with small shunts and those due to the factors already mentioned in the absence of a shunt (Braunwald et al., 1962). A still greater degree of accuracy is obtained by continuous sampling and demonstration of a time difference in the arrival of radioactivity at the shunt chamber and at the chamber immediately upstream; and we have accordingly modified our ${ }^{32} \mathrm{P}$ technique for the use of krypton.

It is still possible, with this technique, to encounter the effects of valvular incompetence. As mentioned above, the combination of a ventricular septal defect with tricuspid incompetence produces a result that would be expected from an atrial septal defect. The correlation of physical signs, routine catheter findings, and the additional information from the ${ }^{85} \mathrm{Kr}$ test enables a full pre-operative diagnosis to be made.

The technique requires elaborate apparatus and careful precautions to avoid leakage of radioactive gas. These are justifiable when investigating a patient in whom routine methods suggest a complicated lesion.

\section{SUMMARY}

The radioactive krypton method for detection of left-to-right shunts has been modified by the introduction of continuous sampling. This enables detection of the premature arrival of radioactive blood through a shunt. 
The subject inhales a single breath of krypton-85, while blood, withdrawn by catheter from different right heart chambers in turn, is continuously monitored by a scintillation flow-counter.

By this technique, radioactivity can be picked up downstream of a left-to-right shunt some 10-12 seconds before its arrival proximal to the defect via the normal circulatory pathway. This assessment of time interval leads to more accurate localization than a comparison of saturations. We found the technique safe and valuable in different problems.

We would like to thank Professor J. Rotblat for the advice and encouragement which he has given to us in this work, and to Dr. Graham Hayward, Mr. O. S. Tubbs, and Mr. I. M. Hill for permission to study patients under their care, and Drs. M. Honey and D. A. Chamberlain for their help with the investigations.

\section{REFERENCES}

Braunwald, E., Goldblatt, A., Long, R T. L., and Morrow, A. G. (1962). The krypton 85 inhalation test for the detection of left-to-right shunts. Brit. Heart J., 24, 41.

Jaimet, C. H., Tomlinson, R. H., and Nace, P. F. (1958). Inhalation Radiography. In 2nd U.N. Int. Conf. on the Peaceful Uses of Atomic Energy, Geneva, 1958, Vol. 26, p. 94.

Long, R. T. L., Braunwald, E., and Morrow, A. G. (1960). Intracardiac injection of radioactive krypton. Circulation, 21, 1126.

Morrow, A. G., Sanders, R. J., and Braunwald, E. (1958). The nitrous oxide test: an improved method for the detection of left-to-right shunts. Circulation, 17, 284.

Sanders, R. J., and Morrow, A. G. (1959). The identification and quantification of left-to-right circulatory shunts: a new diagnostic method utilizing the inhalation of a radioactive gas, $85 \mathrm{Kr}$. Amer. J. Med., 26, 508.

Warren, J. V., Stead, E. A., and Brannon, E. S. (1946). The cardiac output in man: a study of some of the errors in the method of right heart catheterization. Amer. J. Physiol., 145, 458.

Weitzman, D., and McAlister, J. (1958). Tracer method for localizing left-to-right cardiac shunts. Lancet, 2 , 1356. 